\title{
Psychoneuroimmunology of Immune Reconstitution Inflammatory Syndrome (IRIS): The New Frontier in Translational Biomedicine
}

\begin{abstract}
This Editorial presents the position that, in the decade ahead, translational biomedicine will continue to play a premier role in the advancement of patient-centered, evidence-based and effectiveness-focused interventions for ARV-treated HIV/AIDS patients who exhibit IRIS. A well- articulated translational research-effectiveness program in biomedicine is timely and critical not only to develop and obtain better biomarkers of IRIS to understand the physio-pathology of this condition more fully, but also to improve efficient dissemination for increased health literacy of all stakeholders.
\end{abstract}

Keywords: Psychoneuroimmunology (PNI), Immune reconstitution inflammatory syndrome (IRIS), Immunodeficiency virus (HIV), Acquired immune deficiency syndrome (AIDS), Anti-retroviral (ARV) therapy, Recurrent aphthous stomatitis (RAS) ulceration, Translational science, Health literacy, stakeholders

\section{Francesco Chiappelli \\ UCLA School of Dentistry, CHS 63-090, Los Angeles, CA 90095-1768, USA fchiappelli@dentistry.ucla.edu}

Corresponding author:

Francesco Chiappelli

” fchiappelli@dentistry.ucla.edu

UCLA School of Dentistry, CHS 63-090, Los Angeles, CA 90095-1768.

Tel: $310-794-6625$

Fax:310-794-7109

\section{Citation: Chiappelli F.}

Psychoneuroimmunology of Immune Reconstitution Inflammatory Syndrome (IRIS): The New Frontier in Translational Biomedicine. Transl Biomed. 2015, 6:2.

June 10, 2015; Accepted: June 16, 2015; Published: June 19, 2015

\section{Introduction}

The Morbity and Mortality Weekly Report of the Center for Disease Control first reported (June 3, 1981) that five men in Los Angeles had been identified by Michael S. Gottlieb MD and his team with a rare form of pneumonia, which was characteristic of patients with a of weakened immune system $[1,2]$. The patients would eventually die of what became known a year later as the Acquired Immune Deficiency Syndrome (AIDS). This spectrum of conditions was eventually (1983) found, by Gallo's group [3] and Montagnier's group [4] independently, to be consequential to infection by the human immunodeficiency virus (HIV). Since its first report, AIDS has killed close to 50 million women, men, children and elderly world-wide. HIV and its sub-types are believed by most (although the issue is not settled and remains a "vexata questio") to have originated in non-human primates on the African continent [5] centuries ago, and to have "jumped species" in the early/mid 20th century, perhaps as a side-product of colonialism (e.g., bushmeat consumption). What remains unclear is why all epidemic HIV groups emerged in human societies nearly simultaneously in the 20th century, despite very old human exposure to the simian form of HIV. Case/patient "zero" on the American continents seem to have been a Canadian man in the mid/late 1970's (Canadian airline steward named
Gaëtan Dugas, who presented aggressive Kaposi sarcoma in the early 1980's), although it is argued that HIV had spread on the American continent long before.

HIV/AIDS is recognized today as a spectrum of conditions caused by infection with the lentivirus HIV of the family of Retroviridae. HIV is tropic to a subpopulation of actively dividing cells in the human immune system, which are characterized by the cluster of differentiation \#4 (CD4) and the $\alpha$ - chemokine receptor, CXCR4. HIV's that use only the CCR5 receptor are termed R5; those that use only CXCR4 are termed X4, and those that use both, X4R5. People with the CCR5- $\Delta 32$ mutation are resistant to infection with R5 virus, as the mutation hampers HIV binding to its co-receptor and blunts infectivity. HIV-tropic cells include primarily the predominant group of $T$ cells (CD4+ $+^{\text {high }} \mathrm{T}$ cells), as well as some macrophages and dendritic cells, which are typically CD4+low. HIV kills CD4+ cells by a number of mechanisms, including apoptosis of uninfected bystander cells, direct viral killing of infected cells, and killing of infected CD4+ T cells by CD8 cytotoxic lymphocytes that recognize infected cells. When CD4+ $T$ cell numbers decline below a critical level, cell-mediated immunity is compromised, and the body becomes progressively more susceptible to opportunistic infections, including aggressive cancers (e.g., extensive fast-growing Kaposi sarcoma, Hodgkin's 
and non-Hodgkin's lymphomas, leukemias), oral and generalized mucosal pathologies (e.g., hairy leukoplakia), aggressive bacterial infections (e.g., pneumocystis pneumonia), severe weight loss (i.e., cachexia), infection of the central nervous system leading to Neuro-AIDS, and the like [6-8].

\section{Anti-Retroviral (ARV) Therapy and Immune Re- constitution Inflammatory Synd rome (IRIS)}

Great progress has been made in our understanding of HIV/ AIDS disease, and its treatment that is generally directed either at blocking the virus (i.e., anti-retroviral, ARV) or reconstituting the immune system, or both jointly. The goal of ARV therapy in immune-compromised HIV-infected individuals, as first conceived over a decade ago [9], indeed was immune reconstitution.

Nonetheless, concerning serious side effects have been observed in $35-45 \%$ of ARV-treated HIV/AIDS patients, who develop a separate set of symptoms due to generalized uncontrolled inflammation, a condition now referred to as the Immune Reconstitution Inflammatory Syndrome (IRIS). IRIS, also known as immune restoration disease, is pathogen-specific inflammatory response in HIV-infected patients that may be triggered after either initiation or re-initiation of ARV therapy, or a protocol change to a more active ARV therapy. IRIS research is today's new frontier in solving HIV/AIDS.

The development of IRIS can be prevented by starting ARV treatment early and before the CD4+ T cell count drops below 100 cells/ $\mu \mathrm{L}$, or (and) by delaying ARV for 4-8 weeks until any coexisting infection resolves. However, this could be an unethical gamble, which could violate the concept of equipoise, since delaying ARV may place patients at greater risks of progression of AIDS pathologies, which may decrease quality of life and endanger the patient's life. By contrast, wanton initiation of ARV may precipitate patients into IRIS episodes, which can include severe irreversible cytomegalovirus-induced liver failure or retinitis, mycobacterium infection, fungi, virus, and aggressive recurrence of HIV/AIDS cancers (e.g., widespread fast growing Kaposi sarcoma). IRIS involves as well re-emergence of oral manifestations, such as recurrent aphthous stomatitis (RAS), hairy leukoplakia and oral Kaposi, and involve other sequestrated sites, such as the bones. In brief, IRIS leads to a plethora of immune pathologies as early as within 4-8 weeks after initiation of ARV therapy [10-14].

IRIS is also characterized by an increase in CD4 cell count as well as a rapid decrease in viral load. Although most cases of IRIS occur in patients who have low CD4 counts and high viral load levels at the time ARV therapy is initiated, IRIS can occur at any CD4 count.

Minor IRIS presentations may simply require the clinicians to reassure patients that these symptoms indicate beneficial immune reconstitution and will resolve with standard treatment to alleviate inflammation (e.g., nonsteroidal anti-inflammatory agents, drainage of abscesses, excision of painful lymph nodes). Severe IRIS, which can also include CNS-IRIS [15], may threaten a patient's overall functional status by causing permanent disability or even death. ARV may have to be stopped until the IRIS flare-ups are controlled with high dose prednisone and antibiotics, since IRIS is generally self- limiting and only symptomatic treatment is needed, including treating IRIS infection. ARV should be reinstated as soon as possible, lest the scenario be harmful to the patient and life-threatening [10-15].

\section{Psychoneuroimmunology in HIV/AIDS, and IRIS}

Psychoneuroendocrine and cellular immune pathways are cross-regulatory mind-body metabolic processes, and have direct effects on immune surveillance of HIV and the onset and progression of AIDS $[16,17]$. These inter-relationships permeate to the cross-regulation of bone metabolism and cellular immunity (i.e., osteoimmunology, osteo-psychoneuro-immunology) [18].

To be clear, the role of psychoneuroimmunology in IRIS is putative and hypothetical at this stage, but the supporting lines of evidence are compelling. The very fact that about one third of the ARV-treated patients develop IRS is suggestive of certain shared features of immune deregulation among a fairly large cohort of HIV/AIDS patients. The observation that one among the primary features of IRIS is aggressive RAS is indicative that the perception of psycho-emotional stress may play an important role in the IRIS process because the causative factor of stress and anxiety in the onset of RAS and other oral lesions is well documented [19]. Indeed, stress and anxiety have seriously harmful outcomes to the oral cavity, including potentially severe damage to the temporomandibular joint, which can be even more significant in HIV/AIDS patients $[18,20]$

Future research in the biomedical complexities of IRIS must elucidate not only the immunopathogenesis of the syndrome, but also its psychoneuroendocrine interdepence - data seem to suggest a slight preponderance of pre-menopausal HIV/AIDS women developing IRIS compared to age-matched men, which aligns with the evidence of sex disparities in cellular immunity [21]. To be clear, future IRIS research must as well consider the relevance of mind-body therapies to control and contain inflammatory flare-ups, such as mindfulness meditation [22] and others motivational (e.g., motivational interviewing) and stressreduction interventions. Research synthesis and systematic reviews to date support the hypothesis that well-targeted mindbody therapies are effective in reducing markers of inflammation and influence virus-specific immune responses [23].

\section{Conclusion}

Translational Biomedicine for Osteo-Psychoneuro-Immunology in IRIS

Translational biomedicine, viz. translational healthcare, aims to obtain the best evidence base for improving the health of individuals and to utilize it in specific clinical settings in the community for diagnosis, intervention, policies and education. Operationally, the role of translational biomedicine in IRIS research must be articulated along the three distinct interrelated domains of (1) engagement of the stakeholders, (2) dissemination, and (3) increased health literacy of the best evidence base. This program, which constitutes our current research endeavor, is aimed at addressing translational block 1 (Tb1, cf. Institute of Medicine's Clinical Research Roundtable) for obtaining and testing basic research findings in clinical settings 
(i.e., translational research, $\mathrm{NIH}$ ), and $\mathrm{Tb} 2$ for utilizing the best evidence base for interventions in standard clinical practice (i.e., translational effectiveness, AHRQ) [24].

In conclusion, translational biomedicine must continue to play a premier role in the advancement of patient-centered, evidence-based and effectiveness-focused interventions for ARV-treated HIV/AIDS patients who exhibit IRIS. New progress awaits us for improved understanding of how inflammatory flare-ups occur in IRIS, to what extent they might be related with psychological states of anxiety, and consequentially mediated by psychoneuroendocrine processes, and in turn modulate secondary diseases, including osteo-pathologies of the temporomandibular joint. A well-articulated translational research-effectiveness program in biomedicine is timely and critical not only to develop and obtain better biomarkers of IRIS to understand the physio-pathology of this condition fully, but also to ensure increased health literacy among patients [25], caregivers and all stakeholders [26] through powerful and efficient dissemination [27].

\section{Acknowledgements}

The author thanks the Evidence-Based Decisions Active Groups of Stakeholders (EBD-AGS) of the EBD-Practice-Based Research Network (ebd-pbrn.org), and the students and colleagues of the EBD Study Group, including and in particular Dr. Olivia Cajulis for edifying discussion about clinical dentistry, and ambulatory general dentistry care. Funded in part by UCLA Senate grants and Fulbright Specialist grant (5077) to FC. 


\section{References}

Centers for Disease Control (CDC) (1981) Pneumocystis pneumonia-Los Angeles. MMWR Morb Mortal Wkly Rep 30: 250-252.

Gottlieb MS, Schroff R, Schanker HM, Weisman JD, Fan PT, et al. (1981) Pneumocystis carinii pneumonia and mucosal candidiasis in previously healthy homosexual men: Evidence of a new acquired cellular immunodeficiency. New England Journal of Medicine 305: 1425-1431.

Sarngadharan MG, DeVico AL, Bruch L, Schüpbach J, Gallo RC (1984) HTLV-III: the etiologic agent of AIDS. Princess Takamatsu Symp 15: 301-308.

Klatzmann D, Laporte JP, Achour A, Brisson E, Gruest J, et al. (1986) [Functional inhibition by cyclosporin $A$ of the lymphocyte receptor for the AIDS virus (HIV)]. C R Acad Sci III 303: 343-348.

D'arc M, Ayouba A, Esteban A, Learn GH, Boué V, et al. (2015) Origin of the HIV-1 group $\mathrm{O}$ epidemic in western lowland gorillas. Proc Nat Acad Sci U S A 112: E1343-1352.

Vajpayee M, Negi N, Kurapati S (2013) The enduring tale of T cells in HIV immunopathogenesis. Indian J Med Res 138: 682-699.

Patton LL (2013) Oral lesions associated with human immunodeficiency virus disease. Dent Clin North Am 57: 673-698.

Minagar A, Commins D, Alexander JS, Hoque R, Chiappelli F, et al. (2008) NeuroAIDS: characteristics and diagnosis of the neurological complications of AIDS. Mol Diagn Ther 12: 25-43.

Dybul M, Fauci AS, Bartlett JG, Kaplan JE, Pau AK; Panel on Clinical Practices for Treatment of HIV (2002) Guidelines for using antiretroviral agents among HIV-infected adults and adolescents. Ann Intern Med 137: 381-433.

10 Murthy AR, Marulappa R, Hegde U, Kappadi D, Ambikathanaya UK, et al. (2015) Treatment guidelines and prognosis of immune reconstitution inflammatory syndrome patients: a review. J Int Oral Health 7: 92-95.

Ramírez-Amador VA, Espinosa E, González-Ramírez I, Anaya-Saavedra $\mathrm{G}$, Ormsby CE, et al. (2009) Identification of oral candidosis, hairy leukoplakia and recurrent oral ulcers as distinct cases of immune reconstitution inflammatory syndrome. Int J STD AIDS 20:259-261.

Walker NF, Scriven J, Meintjes G, Wilkinson RJ (2015) Immune reconstitution inflammatory syndrome in HIV-infected patients. HIV AIDS (Auckl) 7: 49-64.

3 Ogbuagu O, Villanueva M (2014) Extensive Central Nervous System Cryptococcal Disease Presenting as Immune Reconstitution Syndrome in a Patient with Advanced HIV: Report of a Case and Review of Management Dilemmas and Strategies. Infect Dis Rep 6: 5576.

Espinosa E, Ormsby CE, Vega-Barrientos RS, Ruiz-Cruz M, MorenoCoutiño $G$, et al (2010) Risk factors for immune reconstitution inflammatory syndrome under combination antiretroviral therapy can be aetiology-specific. Int J STD AIDS 21: 573-579.
Post MJ, Thurnher MM, Clifford DB, Nath A, Gonzalez RG, et al (2013). CNS-immune reconstitution inflammatory syndrome in the setting of HIV infection, part 1: overview and discussion of progressive multifocal leukoencephalopathy-immune reconstitution inflammatory syndrome and cryptococcal-immune reconstitution inflammatory syndrome. AJNR Am J Neuroradiol 34:1297-1307

Kiecolt-Glaser JK, Glaser R (1988) Psychological influences on immunity. Implications for AIDS. Am Psychol 43: 892-898.

Cole SW (2008) Psychosocial influences on HIV-1 disease progression: neural, endocrine, and virologic mechanisms. Psychosom Med 70: 562-568.

Barkhordarian A, Ajaj R, Ramchandani MH, Demerjian G, Cayabyab R, et al. (2011) Osteoimmunopathology in HIV/AIDS: A Translational Evidence-Based Perspective. Patholog Res Int 2011: 359242.

9 Chiappelli F, Cajulis OS (2004) Psychobiologic views on stressrelated oral ulcers. Quintessence Int 35: 223-227.

20 Chiappelli F, Bakhordarian A, Bach Q, Demerjian GG (2015) Translational Psychoneuroimmunology in Oral Biology \& Medicine. Forum on Immunopathological Diseases and Therapeutics.

Trigunaite A, Dimo J, Jørgensen TN (2015) Suppressive effects of androgens on the immune system. Cell Immunol 294: 87-94.

Chiappelli F, Barkhordarian A, Demerjian G, Bach Q (2015) Cluster Randomized Stepped Wedge Blinded Controlled Trials (CRSWBCT) In Comparative Effectiveness Research (CER) - Part II: Implications for Temporomandibular Joint Disorders (TMD) Research. Translational Medicine - OMICS 5: e130.

Morgan N, Irwin MR, Chung M, Wang C (2014) The effects of mind-body therapies on the immune system: meta-analysis. PLoS One 9: e100903.

4. Chiappelli F (2014) Fundamentals of Evidence-based Health Care and Translational Science. Springer-Verlag, Heidelberg.

Moura Jde A, Costa BC, de Faria RM, Soares TF, Moura EP, et al. (2013) Improving communication skill training in patient centered medical practice for enhancing rational use of laboratory tests: The core of bioinformation for leveraging stakeholder engagement in regulatory science. Bioinformation 9: 718-720.

6 Barkhordarian A, Demerjian G, Jan A, Sama N, Nguyen M (2015) Stakeholder engagement analysis - a bioethics dilemma in patient-targeted intervention: patients with temporomandibular joint disorders. J Transl Med 13:15

Barkhordarian A, Ramchandani MH, Dousti M, Kelly-Gleason L, Chiappelli $F$ (2012) Disseminating the best available evidence: New challenges in public reporting of health care. Bioinformation 8: 293-295. 